\title{
Multiple stationary solutions and global stabilization of reaction-diffusion Gilpin-Ayala competition model under event-triggered impulsive control
}

\author{
Ruofeng Rao ${ }^{\mathrm{a}}$ \\ ${ }^{a}$ Department of Mathematics, Chengdu Normal University, Chengdu , 611130, China
}

\begin{abstract}
In this paper, the author utilizes Saddle Theorem and variational methods to deduce existence of at least six stationary solutions for reaction-diffusion Gilpin-Ayala competition model (RDGACM). To obtain the global stabilization of the positive stationary solution of the RDGACM, the author designs a suitable impulsive event triggered mechanism (IETM) to derive the global exponential stability of the the positive stationary solution. It is worth mentioning that the new mechanism can exclude Zeno behavior and effectively reduce the cost of impulse control through event triggering mechanism. Besides, compared with existing literature, the restrictions on the parameters of the RDGACM are relaxed so that the methods used in existing literature can not be applied to the relaxed case of this paper, and so the author makes comprehensive use of Saddle Theorem, orthogonal decomposition of Sobolev space $H_{0}^{1}(\Omega)$ and variational methods to overcome the mathematical difficulty. Numerical examples show the effectiveness of the methods proposed in this paper.
\end{abstract}

Keywords: reaction-diffusion Gilpin-Ayala competition model (RDGACM); Saddle Theorem; orthogonal decomposition of Sobolev space; the second eigenvalue of Laplacian operator; impulsive event-triggered mechanism

\section{Introduction: Can the restrictions on of RDGACM be relaxed ?}

Since Gilpin and Ayala found in 1973 that the common ecosystem model did not match a series of experimental data well( [12]), Gilpin-Ayala competition model has been widely studied. Particularly, reaction-diffusion ecosystem models can better reflect the real ecology in the nature (see [4,5,13-17]). And so the reaction-diffusion Gilpin-Ayala competition model (RDGACM) is investigated in this paper. However, in existing results, $\lambda_{1}$ is usually used to control the parameters of RDGACM, for example, [4, Theorem 3.2], [5, (H2)], so that the stability of RDGACM can be ensured, where $\lambda_{1}>0$ is the first positive eigenvalue of the Laplacian operator $-\Delta$ in the Sobolev space $H_{0}^{1}(\Omega)$. It inspires the author to write this paper. In fact, in this paper, the author considers using a bigger positive number $\lambda_{2}$ instead of $\lambda_{1}$, but it will bring a mathematical difficulty to judge the existence of multiple stationary solutions and the stability of RDGACM. Indeed, the author will employ a saddle theorem, orthogonal decomposition of Sobolev space $H_{0}^{1}(\Omega)$ and variational methods to deal with the difficulty. Of course, it becomes one of innovations of this paper.

Email address: ruofengrao@163.com; ruofengrao@cdnu.edu.cn (Ruofeng Rao) 
On the other hand, the author is to reduce the impulse frequency to stabilize globally RDGACM with respect to its positive stationary solution. In existing literature ([4,5]), the impulse instants are fixed, and in this paper, a new impulsive event-triggered mechanism (IETM) will be designed to stabilize globally RDGACM. Of course, the IETM will not only exclude the Zeno behaviour, but also the impulse occurs only when the event is triggered so that it reduces the cost of the unnecessary impulse.

Motivated by some ideas and methods of [1-8, 18-21], the author makes a comprehensive utilization of the critical theory, variational methods and impulse control technique to derive the existence theorem of multiple stationary solutions and the global stabilization of a positive stationary solution for RDGACM. In Section 2, models descriptions and preparatory knowledge are presented. And existence theorem of multiple stationary solutions and global exponential stabilization criterion are derived in Section 3. Furthermore, two explicit numerical examples are given in Section 4 to illuminate the effectiveness of the obtained results. Finally, some conclusions and further considerations are proposed in the last section.

This paper has the following innovations:

$\star$ This paper offers the existence of six stationary solutions of RDGACM against three stationary solutions in [4, Theorem 3.1] (see Remark 8 for details).

$\star$ This paper involves the second eigenvalue $\lambda_{2}$, which expanded the parameters range of RDGACM against the existing results ([4, Theorem 3.1-3.2], [5, Theorem 3.2]).

$\star$ This paper involves the comprehensive applications of Saddle theorem, decomposition of eigenfunctions subspace in $H_{0}^{1}(\Omega)$ and designing impulsive event-triggered mechanism, which means an innovation in methods.

$\star$ In this paper, the newly designed impulsive event-triggered mechanism reduces the cost of impulse control, against the fixed impulsive instants mechanism in [4,5] and the related references therein (see Remark 12 for details).

$\star$ There is a difference between this paper and [2, Statement 2] with respect to applying the same saddle theorem, for the norm of $H_{0}^{1}(\Omega)$ defined in this paper is simpler than that of the proof of [2, Statement 2] so that the methods used in this paper is better than that of [2] .

\section{Preliminaries}

Consider the following reaction-diffusion Gilpin-Ayala competition model (RDGACM):

$$
\left\{\begin{aligned}
\frac{\partial u_{1}}{\partial t} & =d_{1} \Delta u_{1}+u_{1}\left(b_{1}-a_{11} u_{1}^{\theta_{1}}-a_{12} u_{2}\right), \quad t \geqslant 0, x \in \Omega, \\
\frac{\partial u_{2}}{\partial t} & =d_{2} \Delta u_{2}+u_{2}\left(b_{2}-a_{21} u_{1}-a_{22} u_{2}^{\theta_{2}}\right), \quad t \geqslant 0, x \in \Omega, \\
u_{1}(t, x) & =u_{2}(t, x)=0, \quad t \geqslant 0, x \in \partial \Omega, \\
u_{1}(0, x) & =\xi_{1}(x), \quad u_{2}(0, x)=\xi_{2}(x), \quad x \in \Omega,
\end{aligned}\right.
$$

where $\Omega$ is a bounded domain in $\mathbb{R}^{N}(1 \leqslant N \leqslant 3)$ with smooth boundary $\partial \Omega$, For $i=1,2, u_{i}(t, x)$ represents the population density of the $i$ th population at time $t$ and the spatial location $x, b_{i}>0$ represents the birth rate of the 
population of the $i$ th species, and $a_{i j}>0$ represents the competition parameter between the species $i$ and the species j. $d_{i}>0$ represents the diffusion coefficient for the species $i$. Initial value function $\xi_{i}(x)>0$ is bounded for $i=1,2$, $u(t, x)=\left(u_{1}(t, x), u_{2}(t, x)\right)$ is called a positive solution of the ecosystem (2.1) if $u_{i}(t, x) \geqslant 0$ for all $t \geqslant 0$ and $x \in \Omega$, $i=1,2$.

Assume that $u_{*}(x)=\left(u_{1}^{*}(x), u_{2}^{*}(x)\right)^{T}$ is a positive stationary solution of the system $(2.1)$. Set

$$
\left\{\begin{array}{c}
U_{1}=u_{1}-u_{1}^{*}(x) \\
U_{2}=u_{2}-u_{2}^{*}(x)
\end{array}\right.
$$

and the stationary solution $\left(u_{1}^{*}(x), u_{2}^{*}(x)\right)^{T}$ of the system $(2.1)$ corresponds to the zero solution $(0,0)^{T}$ of the following system:

$$
\left\{\begin{array}{c}
\frac{\partial U_{1}}{\partial t}=d_{1} \Delta U_{1}+b_{1} U_{1}-\Phi_{1}\left(U_{1}, U_{2}\right), \quad t \geqslant 0, x \in \Omega, \\
\frac{\partial U_{2}}{\partial t}=d_{2} \Delta U_{2}+b_{2} U_{2}-\Phi_{2}\left(U_{1}, U_{2}\right), \quad t \geqslant 0, x \in \Omega \\
U_{1}(t, x)=U_{2}(t, x)=0, \quad t \geqslant 0, x \in \partial \Omega, \\
U_{1}(0, x)=\eta_{1}(x), \quad U_{2}(0, x)=\eta_{2}(x), \quad x \in \Omega,
\end{array}\right.
$$

where we denote $U=\left(U_{1}, U_{2}\right)^{T}, \eta_{i}(x)=\xi_{i}(x)-u_{i}^{*}(x)$ and

$$
\begin{aligned}
& \Phi_{1}(U)=\left(U_{1}+u_{1}^{*}(x)\right)\left[a_{11}\left(U_{1}+u_{1}^{*}(x)\right)^{\theta_{1}}+a_{12}\left(U_{2}+u_{2}^{*}(x)\right)\right]-u_{1}^{*}(x)\left(a_{11} u_{1}^{*}(x)^{\theta_{1}}+a_{12} u_{2}^{*}(x)\right), \\
& \Phi_{2}(U)=\left(U_{2}+u_{2}^{*}(x)\right)\left[a_{21}\left(U_{1}+u_{1}^{*}(x)\right)+a_{22}\left(U_{2}+u_{2}^{*}(x)\right)^{\theta_{2}}\right]-u_{2}^{*}(x)\left(a_{21} u_{1}^{*}(x)+a_{22} u_{2}^{*}(x)^{\theta_{2}}\right) .
\end{aligned}
$$

The following system is the system (2.3) in form of vector-matrix:

$$
\left\{\begin{array}{l}
\frac{\partial U}{\partial t}=D \Delta U+B U-\Phi(U), \quad t \geqslant 0, \quad x \in \Omega, \\
U(t, x)=0, \quad t \geqslant 0, x \in \partial \Omega, \\
U(0, x)=\eta(x), \quad x \in \Omega,
\end{array}\right.
$$

where $\eta=\left(\eta_{1}, \eta_{2}\right)^{T}$ with $\eta(x)=\xi(x)-u_{*}(x)$ and $\xi=\left(\xi_{1}, \xi_{2}\right)^{T}, U=\left(U_{1}, U_{2}\right)^{T}, \Phi(U)=\left(\Phi_{1}(U), \Phi_{2}(U)\right)^{T}$ and

$$
D=\left(\begin{array}{cc}
d_{1} & 0 \\
0 & d_{2}
\end{array}\right), B=\left(\begin{array}{cc}
b_{1} & 0 \\
0 & b_{2}
\end{array}\right) \text {. }
$$

Now we consider applying impulse control on the system (2.5):

$$
\left\{\begin{array}{l}
\frac{\partial U(t, x)}{\partial t}=D \Delta U+B U-\Phi(U), \quad t \geqslant 0, t \neq t_{k} \quad x \in \Omega \\
U\left(t_{k}, x\right)=H_{k} U\left(t_{k}^{-}, x\right), \quad k \in \mathbb{Z}_{+}, \quad x \in \Omega \\
U(t, x)=0, \quad t \geqslant 0, x \in \partial \Omega \\
U(0, x)=\eta(x), \quad x \in \Omega
\end{array}\right.
$$

where the sequence $\left\{t_{k}, k \in \mathbb{Z}_{+}\right\}$is the set of impulse instants. $\frac{\partial U(t, x)}{\partial t}$ denotes the right-hand derivative of $U(t, x)$ on the time variable $t$, and $H_{k}$ is a constant matrix for $k \in \mathbb{Z}_{+}$. The sequence $\left\{t_{k}, k \in \mathbb{Z}_{+}\right\}$is the set of impulse instants. The 
state $U(t, x)$ is assumed to be right continuous and to have left limits on the time variable $t$. Since the zero solution of the system (2.7) is corresponding to the positive stationary solution $u^{*}(x)$, below we only need to consider the stabilization of the zero solution instead of the stability of positive stationary solution $u^{*}(x)$.

In this paper, the following assumptions are proposed:

(A1) For $i=1,2$, set $0<\theta_{i}=\frac{\hat{\theta}_{i}}{\check{\theta}_{i}}<1$ with $\hat{\theta}_{i}$ being an even number, and $\check{\theta}_{i}$ being an odd number.

(A2)For $i=1,2$, there exist positive constants $M_{i}(i=1,2)$ such that

$$
0 \leqslant u_{1} \leqslant M_{1}, \quad 0 \leqslant u_{2} \leqslant M_{2}
$$

Remark 1. Based on some experimental results, the assumptions $0<\theta_{i}<1$ in (A1) are reasonable (see [4,5, 9-11] for details).

Remark 2. In reality, because the natural resources are limited, it is reasonable to assume a bounded population density $u_{i}$ in the assumption (A2).

Definition 1: Given an impulse instant sequence $\left\{t_{k}, k \in \mathbb{Z}_{+}\right\}$, the stationary solution $\left(u_{1}^{*}(x), u_{2}^{*}(x)\right)^{T}$ is globally exponentially stability if the system (2.7) is globally exponentially stability. And the system (2.7) is globally exponentially stability if there exist positive constants $\lambda>0$ and $\varrho_{i}<1(i=1,2)$ such that for every initial condition $\left(t_{0}, \eta\right)$ with $\left|\eta_{i}\right| \leqslant \varrho_{i} M_{i}(i=1,2)$, the solution satisfies

$$
\|U(t, x)\|_{L^{2}(\Omega)} \leqslant \beta_{0}\|\eta\|_{L^{2}(\Omega)} e^{-\lambda\left(t-t_{0}\right)}, \quad \forall t \geqslant t_{0},
$$

where $\beta_{0}>1$ is a positive number.

Lemma 2.1. ([1]) Let $H=H_{1} \oplus H_{2}$ be a Banach space, and $H_{1}$ is a finite dimension subspace. If $J \in C^{1}(H, \mathbb{R})$, satisfying $J(0)=0$, the (PS) condition. Besides, for some $\delta>0$, the following conditions hold,

(1) $J(u) \leqslant 0$ if $u \in H_{1}$ with $\|u\| \leqslant \delta$;

(2) $J(u) \geqslant 0$ if $u \in H_{2}$ with $\|u\| \leqslant \delta$;

(3) $J$ is bounded below, satisfying $\inf _{H} J<0$,

then $J$ owns at least two non-zero critical points.

Remark 3. The so-called (PS) condition may be found in [2, Definition 2].

Lemma 2.2. ([5, Theorem 3.1]) Set $u^{*}(x)=\left(u_{1}^{*}(x), u_{2}^{*}(x)\right)^{T}$. Suppose the condition (A2) holds, and $0<\theta_{i}<1$ for $i=1,2$. If, in addition, there exists a positive constant $c>0$ such that

$$
0 \leqslant g\left(u^{*}(x)\right) \leqslant c D E
$$

then the ecosystem (2.1) possesses at least a positive bounded stationary solution $\left(u_{1}^{*}(x), u_{2}^{*}(x)\right)$, where $E=(1,1)^{T}$, $g(u)=\left(g_{1}\left(u_{1}, u_{2}\right), g_{2}\left(u_{1}, u_{2}\right)\right)^{T}$ with $u=\left(u_{1}, u_{2}\right)^{T}$ and

$$
g_{1}\left(u_{1}, u_{2}\right)=u_{1}\left(b_{1}-a_{11} u_{1}^{\theta_{1}}-a_{12} u_{2}\right), \quad g_{2}\left(u_{1}, u_{2}\right)=u_{2}\left(b_{2}-a_{21} u_{1}-a_{22} u_{2}^{\theta_{2}}\right),
$$




$$
D=\left(\begin{array}{cc}
d_{1} & 0 \\
0 & d_{2}
\end{array}\right)^{T}>0 .
$$

Remark 4. Lemma 2.2 is the immediate corollary of [5, Theorem 3.1] or derived from [2, Theorem 3.1].

Notations : $\mathbb{Z}_{+}$represents the set of all positive integers, and $*$ denotes the symmetric block in one symmetric matrix. Denote $t_{0}=0$. Let $\lambda_{\max }(A)$ denote the maximum eigenvalue of $A$, and $A \geqslant 0(A \leqslant 0)$ denote the matrix $A$ is a symmetric and positive (negative) semi-definite matrix. The notation $A^{T}$ and $A^{-1}$ denote the transpose and the inverse of $A$, respectively. $C(J, S)=\{\phi: J \rightarrow S$ is continuous $\}$. Denote by $\lambda_{k}$ the $k$ th positive eigenvalue of the operator $-\Delta$ in the Sobolev space $H_{0}^{1}(\Omega)$ equipped with the norm $\|v\|=\sqrt{\int_{\Omega}|\nabla v|^{2} d x}$ for any $v(x) \in H_{0}^{1}(\Omega)$. Particularly, $\lambda_{1}$ and $\lambda_{2}$ are the first and the second positive eigenvalue of the operator $-\Delta$, satisfying $0<\lambda_{1}<\lambda_{2}$. Denote by $E\left(\lambda_{k}\right)$ the eigenfunction space of $\lambda_{k}$, and $H_{0}^{1}(\Omega)=E\left(\lambda_{1}\right) \oplus E\left(\lambda_{1}\right)^{\perp}$, where $E\left(\lambda_{1}\right)^{\perp}=E\left(\lambda_{2}\right) \oplus E\left(\lambda_{3}\right) \oplus \cdots$. Besides, for a vector $U(x)=\left(U_{1}(x), U_{2}(x)\right)^{T} \in \mathbb{R}^{2}$ with $x \in \Omega$, we denote $\|U\|_{L^{2}(\Omega)}=\sqrt{\int_{\Omega}\left(U_{1}^{2}+U_{2}^{2}\right) d x}$, and denote $|U|=\left(\left|U_{1}\right|,\left|U_{2}\right|\right)^{T}$. Particularly, the vector $U \geqslant 0$ represents $U_{i} \geqslant 0$ for $i=1,2$. Denote by $\prod_{i=1}^{n} \zeta_{i}=\zeta_{1} \zeta_{2} \cdots \zeta_{n}$ the product of $n$ real numbers $\zeta_{1}, \zeta_{2}, \cdots, \zeta_{n}$, and by $\Pi_{i=1}^{\infty} \zeta_{i}=\zeta_{1} \zeta_{2} \cdots$ the product of infinitely many real numbers $\zeta_{1}, \zeta_{2}, \cdots$.

Remark 5. Equivalence of norms in finite dimensional spaces yields that there exist two positive constants $\mu_{1}, \mu_{2}$ such that

$$
\mu_{1} \int_{\Omega}|\nabla \omega|^{2} d x \leqslant \int_{\Omega}|\omega(x)|^{2} d x \leqslant \mu_{2} \int_{\Omega}|\nabla \omega|^{2} d x, \quad \forall \omega \in E\left(\lambda_{1}\right)
$$

\section{Main resuts}

Theorem 3.1. Suppose the conditions (A1), (A2) and (2.10) hold. If, in addition, the following two conditions hold:

$$
\begin{aligned}
& d_{1} \lambda_{1}<b_{1}<d_{1} \lambda_{2}, \\
& d_{2} \lambda_{1}<b_{2}<d_{2} \lambda_{2},
\end{aligned}
$$

then the system (2.1) possesses at least six stationary solutions, including the positive solution $\left(u_{1}^{*}(x), u_{2}^{*}(x)\right)^{T}$.

To complete the proof of Theorem 3.1, we need to present the following technique lemmas at first.

Proposition 1. Under the conditions (A1) and (3.1), there are at least two nonzero stationary solutions $\left(\alpha_{1}(x), 0\right)$ and $\left(\alpha_{2}(x), 0\right)$ for the system $(2.1)$.

Proof. Let $(\alpha(x), 0)^{T}$ be a stationary solution of the system (2.1), satisfying

$$
d_{1} \Delta \alpha(x)+\alpha(x)\left(b_{1}-a_{11} \alpha(x)^{\theta_{1}}-a_{12} \cdot 0\right)=0, \quad x \in \Omega ;\left.\quad \alpha(x)\right|_{\partial \Omega}=0,
$$

whose functional is

$$
\Psi(\alpha)=\frac{1}{2} \int_{\Omega}|\nabla \alpha(x)|^{2} d x-\frac{b_{1}}{2 d_{1}} \int_{\Omega}|\alpha(x)|^{2} d x+\frac{a_{11}}{\left(2+\theta_{1}\right) d_{1}} \int_{\Omega} \alpha(x)^{2+\theta_{1}} d x,
$$


It is obvious that $\Psi(0)=0$ and $\Psi \in C^{1}\left(H_{0}^{1}(\Omega), \mathbb{R}^{1}\right)$, and then a critical point of the functional $\Psi$ is corresponding to the solution of the equation (3.3).

Next, we claim that $\Psi$ satisfies the (PS) condition.

Indeed, if there exists $\left\{\alpha_{n}\right\} \subset H_{0}^{1}(\Omega)$, satisfying

$$
\Psi\left(\alpha_{n}\right) \rightarrow a \in \mathbb{R}^{1}
$$

and

$$
\left\|\Psi^{\prime}\left(\alpha_{n}\right)\right\|_{\left(H_{0}^{1}(\Omega)\right)^{*}} \rightarrow 0
$$

which means that when $n$ is big enough,

$$
\psi\left(\alpha_{n}\right)=\frac{1}{2} \int_{\Omega}\left|\nabla \alpha_{n}(x)\right|^{2} d x-\frac{b_{1}}{2 d_{1}} \int_{\Omega}\left|\alpha_{n}(x)\right|^{2} d x+\frac{a_{11}}{\left(2+\theta_{1}\right) d_{1}} \int_{\Omega} \alpha_{n}(x)^{2+\theta_{1}} d x=a+o(1),
$$

Let

$$
\alpha_{n}=\overline{\alpha_{n}}+\widetilde{\alpha_{n}}, \quad \overline{\alpha_{n}} \in E\left(\lambda_{1}\right), \widetilde{\alpha_{n}} \in E\left(\lambda_{1}\right)^{\perp},
$$

then

$$
\begin{aligned}
& \int_{\Omega}\left|\nabla \alpha_{n}(x)\right|^{2} d x-\frac{b_{1}}{d_{1}} \int_{\Omega}\left|\alpha_{n}(x)\right|^{2} d x \\
\geqslant & \left(1-\frac{b_{1}}{d_{1} \lambda_{1}}\right) \int_{\Omega}\left|\nabla \overline{\alpha_{n}}(x)\right|^{2} d x+\left(1-\frac{b_{1}}{d_{1} \lambda_{2}}\right) \int_{\Omega}\left|\nabla \widetilde{\alpha_{n}}(x)\right|^{2} d x
\end{aligned}
$$

The condition (A1) yields there is $c_{1}>0$ big enough such that

$$
\int_{\Omega} \alpha_{n}(x)^{2+\theta_{1}} d x \geqslant \int_{\Omega}\left(c_{1}\left|\overline{\alpha_{n}}(x)\right|^{2}+c_{1}\left|\widetilde{\alpha_{n}}(x)\right|^{2}-c_{2}\right) d x
$$

where $c_{2}$ is a constant dependent upon $c_{1}$.

It follows by (3.7) that

$$
\begin{aligned}
& \quad\left[\left(c_{1} \mu_{1} \frac{a_{11}}{\left(2+\theta_{1}\right) d_{1}}+\frac{1}{2}\left(1-\frac{b_{1}}{d_{1} \lambda_{1}}\right)\right) \int_{\Omega}\left|\nabla \overline{\alpha_{n}}(x)\right|^{2} d x+\frac{1}{2}\left(1-\frac{b_{1}}{d_{1} \lambda_{2}}\right) \int_{\Omega}\left|\nabla \widetilde{\alpha_{n}}(x)\right|^{2} d x\right]-c_{2} \frac{a_{11}}{\left(2+\theta_{1}\right) d_{1}} m e s(\Omega) \\
& \leqslant a+1+|a|,
\end{aligned}
$$

where $c_{1}$ is a positive number such that $\alpha(x)^{2+\theta_{1}} \geqslant c_{1}|\alpha(x)|^{2}-c_{2}$ with $c_{1} \mu_{1} \frac{a_{11}}{\left(2+\theta_{1}\right) d_{1}}+\frac{1}{2}\left(1-\frac{b_{1}}{d_{1} \lambda_{1}}\right)>0$, and $c_{2}$ is a constant dependent upon $c_{1}$. And (3.9) implies there exists a constant $c_{3}>0$ such that

$$
c_{3}\left\|\alpha_{n}(x)\right\|^{2} \leqslant a+1+|a|+c_{2} \frac{a_{11}}{\left(2+\theta_{1}\right) d_{1}} \operatorname{mes}(\Omega),
$$

which verifies the boundedness of $\left\{\alpha_{n}\right\}$ in the Sobolev space $H_{0}^{1}(\Omega)$. Further, obviously there exist two positive numbers $c_{4}, c_{5}>0$ big enough such that

$$
\left|\frac{b_{1}}{d_{1}} \alpha(x)-\frac{a_{11}}{d_{1}} \alpha(x)^{1+\theta_{1}}\right|<c_{4}+c_{5}|\alpha(x)|^{3}, \quad \forall(x, \alpha) \in \Omega \times \mathbb{R}^{1}, \Omega \subset R^{N}(1 \leqslant N \leqslant 3),
$$


which means the Caratheodory condition is satisfied. Employing the methods used in the proof of [2, Statement 2] or [3, Theorem 1] results in the existence of a convergent subsequence of the bounded sequence $\left\{\alpha_{n}\right\}$ in the Sobolev space $H_{0}^{1}(\Omega)$, and hence the (PS) condition is satisfied.

If $\alpha \in E\left(\lambda_{1}\right)$ with $\|\alpha\| \leqslant \delta$, then Sobolev embedding theorem or equivalence of norms in finite dimensional spaces yields that there is a constant $c_{6}>0$ such that

$$
\begin{aligned}
\Psi(\alpha) & =\frac{1}{2} \int_{\Omega}|\nabla \alpha(x)|^{2} d x-\frac{b_{1}}{2 d_{1}} \int_{\Omega}|\alpha(x)|^{2} d x+\frac{a_{11}}{\left(2+\theta_{1}\right) d_{1}} \int_{\Omega} \alpha(x)^{2+\theta_{1}} d x \\
& \leqslant-\frac{1}{2}\left(\frac{b_{1}}{d_{1} \lambda_{1}}-1\right)\|\alpha\|^{2}+c_{6} \frac{a_{11}}{\left(2+\theta_{1}\right) d_{1}}\|\alpha\|^{2+\theta_{1}},
\end{aligned}
$$

which implies that there exists $\delta>0$ small enough such that $\Psi(\alpha) \leqslant 0$.

If $\alpha \in E\left(\lambda_{1}\right)^{\perp}$ with $\|\alpha\| \leqslant \delta$, then

$$
\begin{aligned}
\Psi(\alpha) & =\frac{1}{2} \int_{\Omega}|\nabla \alpha(x)|^{2} d x-\frac{b_{1}}{2 d_{1}} \int_{\Omega}|\alpha(x)|^{2} d x+\frac{a_{11}}{\left(2+\theta_{1}\right) d_{1}} \int_{\Omega} \alpha(x)^{2+\theta_{1}} d x \\
& \geqslant \frac{1}{2} \int_{\Omega}|\nabla \alpha(x)|^{2} d x-\frac{b_{1}}{2 d_{1}} \int_{\Omega}|\alpha(x)|^{2} d x \geqslant \frac{1}{2}\left(1-\frac{b_{1}}{d_{1} \lambda_{2}}\right)\|\alpha\|^{2} \geqslant 0
\end{aligned}
$$

Finally, we need to prove $\Psi$ is bounded below.

The proof is similar to that of the boundedness of the (PS) sequence $\left\{\alpha_{n}\right\}$. For convenience, we still use the above symbols.

Let

$$
\alpha=\bar{\alpha}+\widetilde{\alpha}, \quad \bar{\alpha} \in E\left(\lambda_{1}\right), \widetilde{\alpha} \in E\left(\lambda_{1}\right)^{\perp}
$$

then

$$
\int_{\Omega}|\nabla \alpha(x)|^{2} d x-\frac{b_{1}}{d_{1}} \int_{\Omega}|\alpha(x)|^{2} d x \geqslant\left(1-\frac{b_{1}}{d_{1} \lambda_{1}}\right) \int_{\Omega}|\nabla \bar{\alpha}(x)|^{2} d x+\left(1-\frac{b_{1}}{d_{1} \lambda_{2}}\right) \int_{\Omega}|\nabla \widetilde{\alpha}(x)|^{2} d x
$$

The condition (A1) yields there is $c_{1}>0$ big enough such that

$$
\int_{\Omega} \alpha(x)^{2+\theta_{1}} d x \geqslant \int_{\Omega}\left(c_{1}|\bar{\alpha}(x)|^{2}+c_{1}|\widetilde{\alpha}(x)|^{2}-c_{2}\right) d x
$$

where $c_{2}$ is a constant dependent upon $c_{1}$.

It follows by (3.4) that

$$
\begin{aligned}
\psi(\alpha) \geqslant & {\left[\left(c_{1} \mu_{1} \frac{a_{11}}{\left(2+\theta_{1}\right) d_{1}}+\frac{1}{2}\left(1-\frac{b_{1}}{d_{1} \lambda_{1}}\right)\right) \int_{\Omega}|\nabla \bar{\alpha}(x)|^{2} d x+\frac{1}{2}\left(1-\frac{b_{1}}{d_{1} \lambda_{2}}\right) \int_{\Omega}|\nabla \widetilde{\alpha}(x)|^{2} d x\right] } \\
& +\frac{a_{11}}{\left(2+\theta_{1}\right) d_{1}} \int_{\Omega}\left(c_{1}|\widetilde{\alpha}(x)|^{2}-c_{2}\right) d x \\
\geqslant & -c_{2} \frac{a_{11}}{\left(2+\theta_{1}\right) d_{1}} \operatorname{mes}(\Omega),
\end{aligned}
$$

where $c_{1}$ is a positive number such that $\alpha(x)^{2+\theta_{1}} \geqslant c_{1}|\alpha(x)|^{2}-c_{2}$ with $c_{1} \mu_{1} \frac{a_{11}}{\left(2+\theta_{1}\right) d_{1}}+\frac{1}{2}\left(1-\frac{b_{1}}{d_{1} \lambda_{1}}\right)>0$, and $c_{2}$ is a constant dependent upon $c_{1}$. Thus, (3.19) verifies that $\Psi$ is bounded below. 
Now, completely similar as the proof of [2, Statement 2], we can similarly prove that if $\inf _{H_{0}^{1}(\Omega)} \Psi \geqslant 0$ implies infinitely many positive stationary solutions and infinitely many negative stationary solutions for the ecosystem (2.1), and if $\inf _{H_{0}^{1}(\Omega)} \Psi<0$, Lemma 2.1 means two non-zero critical points for $\Psi$, which completes the proof.

Remark 6. In [4, Theorem 3.1], the restriction conditions $b_{i}<d_{i} \lambda_{1}$ are too harsh. But in Proposition 1, we allow more relaxed conditions $b_{1}<d_{1} \lambda_{2}$. Of course, limited to the length of this article, we only consider the case $d_{1} \lambda_{1}<$ $b_{1}<d_{1} \lambda_{2}$, for the methods similar as that of [4, Theorem 3.1] can deal with the case $b_{1}<d_{1} \lambda_{1}$.

Remark 7. The difference in the conditions pointed out in Remark 6 brings out a mathematical difficulty to this paper. In fact, the restriction conditions $b_{i}<d_{i} \lambda_{1}$ in [4] guarantee the compactness of the (PS) sequences, which does not become true in the case of $b_{i}>d_{i} \lambda_{1}$ in Proposition 1. By utilizing the orthogonal decomposition of the Sobolev space $H_{0}^{1}(\Omega)$ in [6-8], we employ the saddle theorem (Lemma 2.1) to overcome the mathematical difficulty in Proposition 1. Besides, although we are inspired by some methods used in the proof of [2, Statement 2], we can see that the techniques of the proof of Proposition 1 are actually different from those of [2] to some extent, which means that Proposition 1 is a new result.

Similarly, we can deduce the following Proposition.

Proposition 2. Under the condition (A1) and (3.2), there are at least two nonzero stationary solution $\left(0, \beta_{1}(x)\right)$ and $\left(0, \beta_{2}(x)\right)$ for the system $(2.1)$.

Proof of Theorem 3.1. Firstly, zero solution $(0,0)^{T}$ is obviously a trivial solution of the system (2.1). Next, Proposition 1 and Proposition 2 provide four nonzero stationary solution $\left(\alpha_{i}(x), 0\right)$ and $\left(0, \beta_{i}(x)\right)$, where $i=1,2$. The condition (2.10) and Lemma 2.2 implies the existence of positive stationary solution $\left(u_{1}^{*}(x), u_{2}^{*}(x)\right)^{T}$ for the ecosystem (2.1), which is obviously different from the above-mentioned five stationary solution due to the fact $u_{i}^{*}(x)>0$ for all $x \in \Omega$ and $i=1,2$.

Remark 8. The condition (2.10) together with [5, Theorem 3.1] or [2, Theorem 3.1] guarantees that $\alpha_{i}(x) \geqslant 0$ and $\beta_{i}(x) \geqslant 0$ in the proof of Theorem 3.1.

Remark 9. The condition (3.1) $d_{1} \lambda_{1}<b_{1}<d_{1} \lambda_{2}$ is different from the condition $d_{1} \lambda_{1}>b_{1}$ of [4, Theorem 3.2], and so the condition (3.1) of this paper can not achieve the local stability of stationary solution similarly as that of [4, Theorem 3.2]. It is another mathematical difficulty brought by the condition (3.1). In fact, the condition (3.1) makes it difficult to guarantee the convergence of the (PS) sequence $\left\{\alpha_{n}\right\}$ in the proof of Proposition 1 until we employ the orthogonal decomposition technique of Sobolev space $H_{0}^{1}(\Omega)$, which is completely different from the methods used in the proofs of [4, Theorem 3.1] and [2, Statement 2].

Remark 10. Theorem 3.1 illuminates that the positive stationary solution $\left(u_{1}^{*}(x), u_{2}^{*}(x)\right)^{T}$ must be not globally stable. Recently, [5, Theorem 3.3] proposed a global stabilization on the positive stationary solution by way of impulsive control technique with fixed impulse instants. Below, we shall design the event-triggered impulsive control, in which 
impulses only occur when the event which is related to the states of the system is triggered so that the cost of manual control is greatly reduced. This is the innovation of this paper which is different from [5]. But event-triggered impulsive control may brings out Zeno behaviour, and so another difficulty of this paper is to exclude the possibility of Zeno behaviour, for only a well designed impulsive event-triggered mechanism (IETM) can make the possibility eliminated.

Below, we design the impulsive event-triggered mechanism (IETM) as follows:

For a given diagonal matrix $P>0$, and positive constants $\lambda>0$, we define the impulse instants as follows,

$$
t_{k}=\inf \left\{t \geqslant t_{k-1}: \int_{\Omega} U^{T}(t, x) P U(t, x) d x>\zeta_{k} e^{-\lambda\left(t-t_{k-1}\right)} \int_{\Omega} U^{T}\left(t_{k-1}, x\right) P U\left(t_{k-1}, x\right) d x\right\}, \quad k \in \mathbb{Z}_{+},
$$

where $\zeta_{k}>1$, satisfying

$$
\Pi_{i=1}^{+\infty} \zeta_{i}=+\infty
$$

It is obvious that $t_{1}>t_{0}$, for $\int_{\Omega} U^{T}\left(t_{0}, x\right) P U\left(t_{0}, x\right) d x<e^{\left(a_{1}-\lambda\left(t_{0}-t_{0}\right)\right)} \int_{\Omega} U^{T}\left(t_{0}, x\right) P U\left(t_{0}, x\right) d x$.

Below, we begin to consider the global stabilization on the ecosystem under event-triggered impulsive control.

Proposition 3. If there exists positive constant $c_{0}>0$ such that

$$
\frac{\lambda_{\max } \Theta}{\lambda_{\min } P} \leqslant c_{0}
$$

then there does not exist Zeno behaviour under IETM(3.14), where

$$
\Theta=\left(\begin{array}{cc}
-2 \lambda_{1} d_{1}+2 b_{1}+2 a_{11}\left(1+\theta_{1}\right) M_{1}^{\theta_{1}}+2 a_{12} M_{2} & a_{12} M_{1}+a_{21} M_{2} \\
* & -2 \lambda_{1} d_{2}+2 b_{2}+2 a_{22}\left(1+\theta_{2}\right) M_{2}^{\theta_{2}}+2 a_{21} M_{1}
\end{array}\right)
$$

Proof. Let $U(t, x)=U\left(t, x ; t_{0}, \eta\right)$ be the solution the system (2.7) with the initial value $\left(t_{0}, \eta\right)$. Define $v(t)=$ $\int_{\Omega} U^{T}(t, x) P U(t, x) d x$. Let $t_{1}<t_{2}<\cdots<t_{n}<\cdots$ be the trigger instants, and $\mathcal{D}$ be the upper right-hand Dini derivative with respective system (2.7), then Poincare inequality yields

$$
\mathcal{D}^{+} v(t) \leqslant 2 \int_{\Omega}\left[|U|^{T}\left(-\lambda_{1} D+B\right)|U|+|U|^{T}|\Phi(U)|\right] d x
$$

It follows by (2.4), differential mean value theorem and the condition (A2) that

$$
2|U|^{T}|\Phi(U)| \leqslant|U|^{T}\left(\begin{array}{cc}
2 a_{11}\left(1+\theta_{1}\right) M_{1}^{\theta_{1}}+2 a_{12} M_{2} & a_{12} M_{1}+a_{21} M_{2} \\
* & 2 a_{22}\left(1+\theta_{2}\right) M_{2}^{\theta_{2}}+2 a_{21} M_{1}
\end{array}\right)|U|
$$

Combining (3.17),(3.18) and the condition (3.16) results in

$$
\begin{aligned}
\mathcal{D}^{+} v(t) & \leqslant \frac{\lambda_{\max } \Theta}{\lambda_{\min } P} \int_{\Omega}\left|U^{T}\right| P|U| d x \\
& \leqslant c_{0} v(t), \quad t \in\left[t_{k-1}, t_{k}\right), \quad k \in \mathbb{Z}_{+},
\end{aligned}
$$


which together with IETM (3.14) and the condition (3.16) implies that

$$
\zeta_{1} e^{-\lambda\left(t_{1}-t_{0}\right)} v\left(t_{0}\right)=v\left(t_{1}^{-}\right) \leqslant e^{\int_{t_{0}}^{t_{1}} c_{0} d t} v\left(t_{0}\right)=e^{c_{0}\left(t_{1}-t_{0}\right)} v\left(t_{0}\right), \forall n \in \mathbb{Z}_{+},
$$

and hence

$$
t_{1}-t_{0} \geqslant \frac{1}{c_{0}+\lambda} \ln \left(\zeta_{1}\right)
$$

Similarly, we can prove

$$
t_{n}-t_{n-1} \geqslant \frac{1}{c_{0}+\lambda} \ln \left(\zeta_{n}\right)
$$

Hence,

$$
t_{n} \geqslant \frac{1}{c_{0}+\lambda} \ln \left(\Pi_{k=1}^{n} \zeta_{k}\right)+t_{0} \rightarrow+\infty, \quad n \rightarrow \infty
$$

which completes the proof.

Remark 11. [22, Theorem 3.3] illuminates that the inter-execution time intervals of control task must not be arbitrarily small. In the proof of Proposition 3 of this paper, $t_{n}-t_{n-1} \geqslant \frac{1}{c_{0}+\lambda} \ln \left(\zeta_{n}\right)$, but $\frac{1}{c_{0}+\lambda} \ln \left(\zeta_{n}\right)$ may be arbitrarily small, for example, $\zeta_{n}=e^{\frac{1}{n}}>1$ and $\Pi_{n=1}^{\infty} \zeta_{n}=+\infty$. Thus, Proposition 3 is better than some existing result due to its reducing conservatism, for Proposition 3 can also exclude the Zeno behavior.

Theorem 3.2. Under the assumptions of Theorem 3.1 and Proposition 3 , and if, in addition, $H_{k}^{T} P H_{k}>0$ and

$$
\begin{gathered}
0<\gamma_{k}=\frac{\lambda_{\max }\left(H_{k}^{T} P H_{k}\right)}{\lambda_{\min } P}<1, \forall k \in \mathbb{Z}_{+}, \\
\sup _{k \in \mathbb{Z}_{+}} \gamma_{k} \zeta_{k+1}<1,
\end{gathered}
$$

then the impulsive system (2.7) is globally exponentially stable under IETM (3.14). Equivalently, the positive stationary solution $u^{*}(x)$ is globally exponentially stable under IETM (3.14).

Proof. The proof may be completed by considering three cases:

Case 1. If the event is never triggered, IETM (3.14) yields

$$
\int_{\Omega} U^{T}(t, x) P U(t, x) d x \leqslant \zeta_{1} e^{-\lambda\left(t-t_{0}\right)} \int_{\Omega} U^{T}\left(t_{0}, x\right) P U\left(t_{0}, x\right) d x, \quad t \geqslant t_{0} .
$$

Case 2. If the event is triggered in finite times, say $N$ times, we assume $t_{1}<t_{2}<\cdots<t_{N}$ are trigger instants, and IETM (3.14) means

$$
\begin{gathered}
v\left(t_{1}^{-}\right)=\zeta_{1} e^{-\lambda\left(t_{1}-t_{0}\right)} v\left(t_{0}\right), \\
v(t) \leqslant \zeta_{1} e^{-\lambda\left(t-t_{0}\right)} v\left(t_{0}\right), \quad \forall t \in\left[t_{0}, t_{1}\right),
\end{gathered}
$$

and

$$
v(t) \leqslant \zeta_{2} e^{-\lambda\left(t-t_{1}\right)} v\left(t_{1}\right), \quad \forall t \in\left[t_{1}, t_{2}\right) .
$$




$$
v\left(t_{2}^{-}\right)=\zeta_{2} e^{-\lambda\left(t_{2}-t_{1}\right)} v\left(t_{1}\right) .
$$

It follows from (3.20) and (3.23) that

$$
\begin{aligned}
v\left(t_{1}\right) & \leqslant \frac{\lambda_{\max }\left(H_{1}^{T} P H_{1}\right)}{\lambda_{\min }(P)} \int_{\Omega} U^{T}\left(t_{1}^{-}, x\right) P U\left(t_{1}^{-}, x\right) d x \leqslant \gamma_{1} v\left(t_{1}^{-}\right) \\
& \leqslant \gamma_{1} \zeta_{1} e^{-\lambda\left(t_{1}-t_{0}\right)} v\left(t_{0}\right)
\end{aligned}
$$

It follows from (3.27), 3.25) and (3.21) that

$$
\begin{aligned}
v(t) & \leqslant \zeta_{2} e^{-\lambda\left(t-t_{1}\right)} v\left(t_{1}\right) \\
& \leqslant \zeta_{2} e^{-\lambda\left(t-t_{1}\right)} \gamma_{1} \zeta_{1} e^{-\lambda\left(t_{1}-t_{0}\right)} v\left(t_{0}\right) \\
& \leqslant \zeta_{1} e^{-\lambda\left(t-t_{0}\right)} v\left(t_{0}\right), \quad \forall t \in\left[t_{1}, t_{2}\right) .
\end{aligned}
$$

Combining (3.28) and (3.24) results in

$$
v(t) \leqslant \zeta_{1} e^{-\lambda\left(t-t_{0}\right)} v\left(t_{0}\right), \quad \forall t \in\left[t_{0}, t_{2}\right) .
$$

Similarly, we can prove

$$
v(t) \leqslant \zeta_{1} e^{-\lambda\left(t-t_{0}\right)} v\left(t_{0}\right), \quad \forall t \in\left[t_{0}, t_{N}\right),
$$

and

$$
v\left(t_{N}^{-}\right) \leqslant \zeta_{1} e^{-\lambda\left(t_{N}-t_{0}\right)} v\left(t_{0}\right)
$$

Below, we consider the case of $t \geqslant t_{N}$.

Since $t_{N}$ is the last trigger time, IETM (3.14) yields

$$
v(t) \leqslant \zeta_{N+1} e^{-\lambda\left(t-t_{N}\right)} v\left(t_{N}\right), \quad \forall t \geqslant t_{N}
$$

Combining (3.30), (3.31) and (3.21) results in

$$
\begin{aligned}
v(t) & \leqslant \zeta_{N+1} e^{-\lambda\left(t-t_{N}\right)} v\left(t_{N}\right) \\
& \leqslant \zeta_{N+1} e^{-\lambda\left(t-t_{N}\right)} \gamma_{N} v\left(t_{N}^{-}\right) \\
& \leqslant \zeta_{N+1} e^{-\lambda\left(t-t_{N}\right)} \gamma_{N} \zeta_{1} e^{-\lambda\left(t_{N}-t_{0}\right)} v\left(t_{0}\right) \\
& \leqslant \zeta_{1} e^{-\lambda\left(t-t_{0}\right)} v\left(t_{0}\right), \quad \forall t \geqslant t_{N},
\end{aligned}
$$

which together with (3.30) means

$$
v(t) \leqslant \zeta_{1} e^{-\lambda\left(t-t_{0}\right)} v\left(t_{0}\right), \quad \forall t \geqslant t_{0}
$$

Case 3. If the event is triggered in infinite times, we assume $t_{1}<t_{2}<\cdots<t_{k}<\cdots$ are trigger instants. Proposition 3 exclude the Zeno behaviour. Next, for any given $t \geqslant t_{0}$, there exists a $N \in \mathbb{Z}_{+}$such that $t_{N-1} \leqslant t<t_{N}$. By way of the similar discussions in Case 2, we can derive (3.34) for all $t \geqslant t_{0}$, too. That is, (3.34) holds in all cases. Now it follows from (3.34) that 


$$
\begin{aligned}
& \lambda_{\min }(P)\|U(t)\|_{L^{2}(\Omega)}^{2} \leqslant \int_{\Omega} U^{T}(t, x) P U(t, x) d x \leqslant \zeta_{1} e^{-\lambda\left(t-t_{0}\right)} \int_{\Omega} U^{T}\left(t_{0}, x\right) P U\left(t_{0}, x\right) d x \\
\leqslant & \zeta_{1} e^{-\lambda\left(t-t_{0}\right)} \lambda_{\max }(P)\left\|U\left(t_{0}\right)\right\|_{L^{2}(\Omega)}^{2}, \quad t \geqslant t_{0},
\end{aligned}
$$

and hence

$$
\|U(t)\|_{L^{2}(\Omega)}^{2} \leqslant \frac{\lambda_{\max }(P)}{\lambda_{\min }(P)} \zeta_{1} e^{-\lambda\left(t-t_{0}\right)}\|\eta\|_{L^{2}(\Omega)}^{2}, \quad t \geqslant t_{0},
$$

which together with Definition 1 implies that the impulsive system (2.7) is globally exponentially stable under IETM (3.14). Equivalently, the positive stationary solution $u^{*}(x)$ is globally exponentially stable under IETM (3.14).

Remark 12. In Theorem 3.2, the impulse occurs only when the event is triggered. And $t_{n}-t_{n-1} \geqslant \frac{1}{c_{0}+\lambda} \ln \left(\zeta_{n}\right)$ means that if making $\zeta_{n}$ become bigger. Thus, the bigger impulse interval can be guaranteed so that the cost of impulse control is smaller than that of existing results involved in the fixed impulsive interval $($ see $[4,5])$.

\section{Numerical examples}

Example 4.1. Let $\theta_{1}=\frac{2}{3}, \theta_{2}=\frac{4}{5}, b_{i}=0.13+0.0001 i, d_{i}=0.1+0.0001 i, i=1,2$, and $\Omega=(0,4) \times(0,4)$. By way of accurate calculation, we can conclude from Remark 13 that the conditions (3.1) and (3.2) are satisfied. Furthermore, set $M_{i}=2+0.1 i, i=1,2$, and $a_{11}=0.03 ; a_{12}=0.02, a_{21}=0.025 ; a_{22}=0.03$. An accurate calculation can verify that the condition (2.10) is satisfied if letting $c=100000$. Now all the conditions of Theorem 3.1 are satisfied, and so Theorem 3.1 yields that the system (2.1) possesses at least six stationary solutions, including the positive solution $\left(u_{1}^{*}(x), u_{2}^{*}(x)\right)^{T}$.

Remark 13. ([23]) Let $\Omega \subset \mathbb{R}^{n}$, then

$$
\begin{aligned}
& \lambda_{k+1}-\lambda_{k} \leqslant \frac{4 \sum_{i=1}^{k} \lambda_{i}}{n k} \\
& \lambda_{k} \geqslant c_{n}\left(\frac{k}{m e s \Omega}\right)^{\frac{2}{n}},
\end{aligned}
$$

where $c_{n}=\frac{4 \pi^{2}}{\left(\frac{\omega_{n-1}}{n}\right)^{\frac{2}{n}}}, \omega_{n-1}=\operatorname{Area}\left(S^{n-1}\right)$.

Example 4.2. Assume that we use all the data in Example 4.1, then an accurate calculation yields that $\lambda_{1}=1.2337$ (see,e.g. [2, Remark 14]).

$$
\Theta=\left(\begin{array}{ll}
0.2652 & 0.0970 \\
0.0970 & 0.3212
\end{array}\right)
$$

then $\lambda_{\max } \Theta=0.3942$. Moreover, set $P=\operatorname{diag}(1,1)$, and $c_{0}=0.5$, the condition (3.16) is satisfied, and according to Proposition 3, there is not Zeno behaviour under IETM (3.14).

Example 4.3. Assume that we use all the data in Example 4.1 and Example 4.2. Set $H_{k}=\operatorname{diag}(0.7,0.7)$, then an accurate calculation yields that $H_{k}^{T} P H_{k}=\operatorname{diag}(0.49,0.49)>0$ and $\gamma_{k}=\frac{\lambda_{\max }\left(H_{k}^{T} P H_{k}\right)}{\lambda_{\min } P} \equiv 0.49<1$, and then the condition (3.20) is satisfied. 


$$
\sup _{k \in \mathbb{Z}_{+}} \gamma_{k} \zeta_{k+1}=0.49 \times e^{\frac{1}{2}}=0.8079<1
$$

which implies the condition (3.21) is satisfied. Now, all the conditions of Theorem 3.2 are satisfied . Now Theorem 3.2 yeilds that the impulsive system (2.7) is globally exponentially stable under IETM (3.14). Equivalently, the positive stationary solution $u^{*}(x)$ is globally exponentially stable under IETM (3.14).

\section{Conclusions and further considerations}

In this paper, Saddle Theorem, the orthogonal decomposition of Sobolev space $H_{0}^{1}(\Omega)$ and variational methods are applied to deduce the existence of six stationary solutions for RDGACM. Moreover, A new IETM is designed to make the RDGACM stabilized globally. There are many innovations in the ideas and methods (see Remark 6-12 for details). Now, we should consider further work, for example, $d_{1} \lambda_{2}<b_{1}<d_{1} \lambda_{3}$ or $b_{1}>d_{1} \lambda_{3}$. Although there are some inspirations from the orthogonal decomposition technique on eigenfunction spaces (see,e.g.[6,8]), it seems to be an interesting problem.

\section{References}

[1] Haim Brezis, Louis Nirenberg, Remarks on finding critical points, Communications on Pure \& Applied Mathematics 44 (1991) 939 C963.

[2] Ruofeng Rao, Jialin Huang, Xiaodi Li. Stability analysis of nontrivial stationary solution of reaction-diffusion neural networks with time delays under Dirichlet zero boundary value. Neurocomputing, 445C (2021) 105-120.

[3] Xiongrui Wang, Ruofeng Rao, Shouming Zhong. pth Moment Stability of a Stationary Solution for a Reaction Diffusion System with Distributed Delays. Mathematics, 2020, 8, 200.

[4] Ruofeng Rao, Xinsong Yang, Rongqiang Tang, Yulin Zhang, Xinggui Li, Lei Shi. Impulsive stabilization and stability analysis for GilpinAyala competition model involved in harmful species via LMI approach and variational methods. Mathematics and Computers in Simulation, Volume 188, October 2021, Pages 571-590

[5] Ruofeng Rao, Quanxin Zhu, Kaibo Shi. Input-to-State Stability for Impulsive Gilpin-Ayala Competition Model With Reaction Diffusion and Delayed Feedback. IEEE Access, 2020, 8, 222625-222634.

[6] Ruofeng Rao. Existence of multiple nontrivial solutions for the critical elliptic equations $\Delta u=\lambda_{k} u+|u|^{2^{*}-2} u+f(x, u)($ In Chinese). Chinese Annals of Mathematics, Series A, 26(2005), 749-754.

[7] Ruofeng Rao. Ruofeng Rao, On the Elliptic Equations With the First Eigenvalue, Involving the Critial Sobolev Exponents (In Chinese), Advances in Mathematics (China) 33 (6) (2004) 703C711.

[8] Chunlei Tang, Xingping Wu. Existence and Multiplicity of Solutions of Semilinear Elliptic Equations. Journal of Mathematical Analysis and Applications, 2001, Volume 256, Issue 1, Pages 1-12

[9] M. E. Gilpin and F. J. Ayala, Schoener's model and drosophila competition, Theor. Population Biol., vol. 9, no. 1, pp. 12-14, Feb. 1976.

[10] W. R. Thomas, M. J. Pomerantz, and M. E. Gilpin, Chaos, asymmetric growth and group selection for dynamical stability, Ecology, vol. 61, no. 6, pp. 1312-1320, Dec. 1980.

[11] C. M. Moore, S. A. Catella, and K. C. Abbott, Population dynamics of mutualism and intraspecfic density dependence: How $\theta$-logistic density dependence affects mutualistic positive feedback, Ecological Model., vol. 368, pp. 191-197, Jan. 2018.

[12] Gilpin, M.E., Ayala, F.J., Global models of growth and competition. Proceedings of the National Academy of Sciences of the United States of America, 70 (1973) 3590-3593

[13] M. Ma, J. Hu, J. Zhang, J. Tao, A reaction-diffusion model with nonlinearity driven diffusion, Appl. Math. (A Journal of Chinese Universities), 28 (2013) 290-302 . 
[14] N. Shigesada, K. Kawasaki, E. Teramoto, Spatial segregation of interacting species, J. Theore. Bio. 79(17) (1979), 83-99.

[15] Y. Liu, Y. Tao, Dynamics in a parabolic-elliptic two-species population competition model with cross-diffusion for one species, J. Math. Anal. Appl. 456(2017), 1-15.

[16] Ruofeng Rao, Quanxin Zhu, Jialin Huang. Existence, Uniqueness, and Input-to-State Stability of Ground State Stationary Strong Solution of a Single-Species Model via Mountain Pass Lemma. Complexity, Volume 2021, Article ID 8855351. https://doi.org/10.1155/2021/8855351

[17] M. Chen, X. Xie, Bi-stability of two-species competition model with reaction diffusion, J. Huaqiao Univer. (Natural Sci.), 41(2020) 268271.

[18] X. Tan, J. Cao, and X. Li, Consensus of leader-following multiagent systems: A distributed event-triggered impulsive control strategy, IEEE Transactions on Cybernetics, pp. 1-10, 2018.

[19] Y. Zhou and Z. Zeng, Event-triggered impulsive control on quasisynchronization of memristive neural networks with time-varying delays, Neural Networks, vol. 110, pp. 55-65, 2019.

[20] B. Liu, D. J. Hill, and Z. Sun, Stabilisation to input-to-state stability for continuous-time dynamical systems via event-triggered impulsive control with three levels of events, IET Control Theory \& Applications, vol. 12, pp. 1167-1179, 2018.

[21] X. Li and P. Li, Input-to-state stability of nonlinear systems: event-triggered impulsive control. IEEE Transactions on Automatic Control, doi: 10.1109/TAC.2021.3063227.

[22] Xie, W., Zhu, Q. . Input-to-state stability of stochastic nonlinear fuzzy Cohen-Grossberg neural networks with the event-triggered control. International Journal of Control, 2018, 1C17. doi:10.1080/00207179.2018.1540887

[23] Chengtong Qiu, Licha Sun. Lectures on differential geometry. Beijing, Advanced Education Press, 2018. 